\title{
Comparison of Two-Phase Porosity Models for High Capacity Random Pack- ing
}

\author{
Mathias Poulsen ${ }^{1, *}$, Kim Sørensen ${ }^{1,}$, and Thomas Condra ${ }^{1,}$ \\ ${ }^{1}$ AAU energy, Aalborg University, Pontoppidanstræde 111, 9220 Aalborg Ø, Denmark
}

\begin{abstract}
High capacity random packing is used in absorption applications where a large throughput of gas is required while simultaneously maintaining as low a pressure loss as possible. Utilising computational fluid dynamics to capture the internal flow patterns and transients when designing packed bed towers can be advantageous in respect to expected performance and cost optimisation. However, capturing the direct interaction between gas, liquid and packing is not computationally feasible and therefore the packed bed is modelled as a porous media. In this work four different porosity model are calibrated with idealised equations to data for the high capacity packing IMTP or I-Ring. The different models are evaluated based on their ability to predict pressure loss and liquid holdup in the packed bed. An Eulerian two-phase model with a porous zone representing the packed bed is setup in a cylindrical tower. The CFD results are compared to the predictions of the best performing porosity model. It was found that the best performing model had an absolute mean error of $6.7 \%$ when calibrated with the idealised equations. This error increased to $10.5 \%$ when the porosity model was implemented into the CFD model.
\end{abstract}

\section{NOMENCLATURE}

a specific surface area

A viscous model parameter

$\mathscr{A}$ viscous model specific parameter

$B \quad$ inertial model parameter

$\mathscr{B} \quad$ inertial model specific parameter

$C_{h} \quad$ packing specific constant

$d_{p}$ packing diameter

Greek letters

$\alpha$ phase volume fraction

$\eta \quad$ wetting factor

$\overline{\bar{\tau}} \quad$ stress-strain tensor

\section{Subscripts}

dry dry packing

$i$ phase $\mathrm{i}$

$j \quad$ phase $\mathrm{j}$

$g$ gas phase

$l \quad$ liquid phase

$s \quad$ solid phase

wet wetted packing

\section{INTRODUCTION}

Random packed beds are used in various industrial applications where gas-liquid interaction is required such as distillation, gas pollution absorption and $\mathrm{CO}_{2}$ capture. Depending on aspects such as spatial limitations for equipment, power requirements or throughput different types of packing material and geometries are available for making

*e-mail: mps@energy.aau.dk a random packed bed. When a sizeable amount of gas is required to pass through the packed bed and the allowable pressure loss over the packed bed is restricted, high capacity random packing can be used to make up the packed bed. These types of packing materials are characterised by having a porosity above $95 \%$ and a liquid holdup that is below $10-15 \%$ during operation.

In some applications the packed bed is only a part of a tower, and other features may be installed up or down stream of the packed bed. In such cases using computational fluid dynamics to model the tower can give valuable insights about certain conditions during operation. Packing elements often have complex geometric shapes which alters the flow characteristics of both gas and liquid inside the packed bed and therefore no unified model has been developed. Resent studies have modelled small scale towers where the packing material is resolved by the computational mesh, to evaluate specific pressure loss, liquid holdup and interfacial areas $[1,2]$. With the small scale towers requiring mesh sizes in the order of $1.5 \times 10^{6}$ these types of simulations are not suitable for large scale simulations due to the computational resources required. Consequently, modelling of a random packed bed is done by treating it as porous media and thereby reducing the computational requirements significantly.

Depending on the manufacturer the naming of the same packing material differs. The packing material studied in this work is typically referred to by either: IMTP, I-Ring or RSMR. The geometry for this type of packing is shown in Figure 1.

Generally a packing material can be found in different sizes. IMTP can for example be found in sizes varying 


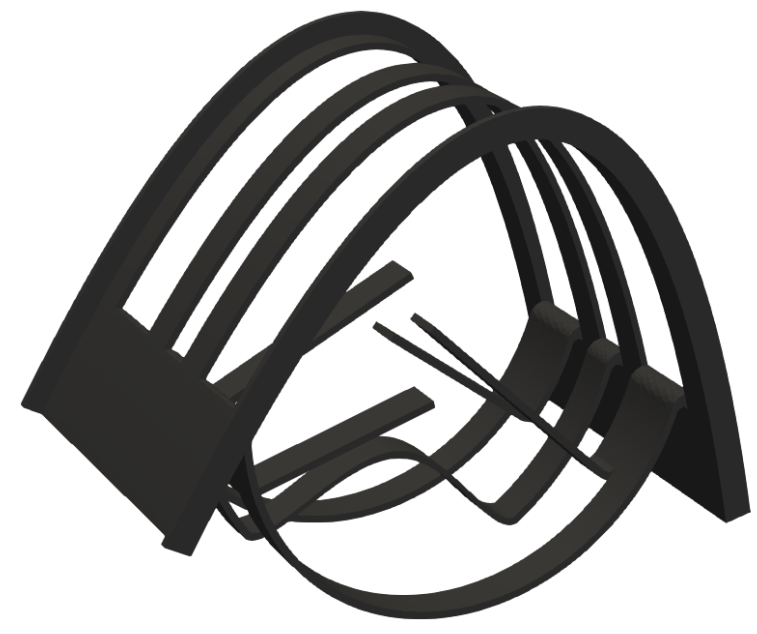

Figure 1. Geometry of IMTP packing material.

from 15 to $70 \mathrm{~mm}$. A smaller packing has a lower bed porosity which leads to a larger liquid holdup and pressure loss at a given gas and liquid load compared to a larger size of the same packing geometry. In the following different porosity models will be compared based on their performance in predicting the pressure loss and liquid holdup in a packed bed consisting of the high performance packing material IMTP-50.

\section{GOVERNING EQUATIONS}

The pressure loss in a packed bed is caused by both by the friction between the gas and packing material and the shear force between the gas and liquid. As more liquid accumulates in the packing the liquid film on the packing elements thickens. This results in both an enlargement of interfacial area between the gas and liquid and a reduction of the cross sectional area in the paths where the gas can pass through the packing, thus leading to an increase in the pressure loss.[3] At some point when the liquid load or the gas velocity is increased the shear force between the gas and liquid becomes large enough to restrict the flow of the liquid film. This causes the liquid holdup to increases considerably which in turn causes the pressure loss to rise. The point of operation where this occurs is termed as the loading point of the packing material [4]. In the following comparison of the different porosity models the operating points considered are below the loading point.

Representing the random packing as a porous media requires a set of closure equations for the Navier-Stokes equations which can represented by a porosity model. In most cases whether it be for single or multi-phase flows the closure equations are some variation of the Ergun equation [5]. Finn and Galvin [6] presented, three different porosity models as candidates for the closure of the Navier-Stokes equiation. The models developed by Attou et al. [7] and Lappalainen et al. [8] are based on solid spherical particles with void fractions in the range of $0.3-0.5$ and liquid holdup in the range 0.2-0.3. The model developed by Fourati et al. [9] is based on parallel slits within a struc- tured packing, where the liquid is assumed to flow as film on the bottom of the slit.

The governing equations for mass and momentum conservation for a given phase in the Eulerian framework without heat and mass transfer can be given as:

$$
\begin{gathered}
\frac{\partial}{\partial t}\left(\alpha_{i} \rho_{i}\right)+\nabla \cdot\left(\alpha_{i} \rho_{i} \vec{u}_{i}\right)=0 \\
\frac{\partial}{\partial t}\left(\alpha_{i} \rho_{i} \vec{u}_{i}\right)+\nabla \cdot\left(\alpha_{i} \rho_{i} \vec{u}_{i} \vec{u}_{i}\right)=-\alpha_{i} \nabla p+\nabla \overline{\bar{\tau}}_{i}+ \\
\alpha_{i} \rho_{i} \vec{g}+\sum \vec{F}_{i j}
\end{gathered}
$$

Finn and Galvin [6], presented a set of idealised steady state, one dimensional versions of Equation 2 for the gas and liquid phase respectively. This set of idealised phase equations can be utilised for calibrating the respective porosity models and are given as:

$$
\begin{gathered}
\alpha_{g} \frac{d p}{d y}+\alpha_{g} \rho_{g} g+F_{l g}+F_{g s}=0 \\
\alpha_{l} \frac{d p}{d y}+\alpha_{l} \rho_{l} g+F_{g l}+F_{l s}=0
\end{gathered}
$$

The interacting forces acting on each phase can be divided into three separate parts that are as follows gas-solid, liquid-solid and gas-liquid. A general expression for each of the interaction forces between the phases can be given as:

$$
\begin{gathered}
\vec{F}_{g s}=(1-\eta)\left(A_{g s}+B_{g s}\left|\vec{u}_{g}\right|\right) \vec{u}_{g} \\
\vec{F}_{l s}=\eta\left(A_{l s}+B_{l s}\left|\vec{u}_{l}\right|\right) \vec{u}_{l} \\
\vec{F}_{g l}=\eta\left(A_{g l}+B_{g l}\left|\vec{u}_{l}-\vec{u}_{g}\right|\right)\left(\vec{u}_{l}-\vec{u}_{g}\right) \\
\vec{F}_{l g}=-\vec{F}_{g l}
\end{gathered}
$$

The wetting factor $\eta$ is an empirical equation correlated from a database of experimental data for various packing types where a packing specific constant $C_{h}$ accounts for the geometric properties of the packing type in question [4]. The wetting factor can be expressed as :

$$
\eta= \begin{cases}C_{h} \operatorname{Re}_{l}^{0.15} F r_{l}^{0.1} & \operatorname{Re}_{l}<5 \\ 0.85 C_{h} \operatorname{Re}_{l}^{0.25} \mathrm{Fr}_{l}^{0.1} & \operatorname{Re}_{l} \geq 5\end{cases}
$$

As it can be seen from Equation 9, the wetting factor is dependent on the liquid Reynolds and Froude number. Both dimensionless groups are based on the superficial velocity and the specific surface area of the packing material. The superficial Reynolds and Froude number can be given as:

$$
R e_{l}=\frac{\left|\alpha_{l} \vec{u}_{l}\right| \rho_{l}}{a_{s} \mu_{l}} \quad F r_{l}=\frac{\left|\alpha_{l} \overrightarrow{u_{l}}\right|^{2} a_{s}}{|\vec{g}|}
$$

By evaluating the gas-solid interaction with data for dry packing material and the liquid-solid interactions only with liquid flowing through the packed bed, Equations 3 and 4 can be reduced to:

$$
\begin{gathered}
\alpha_{g} \frac{d p}{d y}+\alpha_{g} \rho_{g} g+F_{g s}=0 \\
\alpha_{l} \rho_{l} g+F_{l s}=0
\end{gathered}
$$




\section{Porosity models}

The liquid solid interaction force is the same for first three models, and can be given as:

$$
\begin{aligned}
A_{l s} & =\mathscr{A}_{l s} \mu_{l} \frac{\alpha_{s}^{2}}{d_{p}^{2} \alpha_{l}} \\
B_{l s} & =\mathscr{B}_{l s} \rho_{l} \frac{\alpha_{s}}{d_{p}}
\end{aligned}
$$

For the models by Attou et al. [7] and Lappalainen et al. [8] the gas-solid and gas-liquid are described by the same expression. Only the in model by Fourati et al. [9] the gas-liquid interaction has a distinctive expression. The coefficients for the respective models can be given as:

Model 1 - Attou et al. [7]:

$$
\begin{gathered}
A_{g j}=\mathscr{A}_{g j} \mu_{g} \frac{\left(1-\alpha_{g}\right)}{d_{p}^{2} \alpha_{g}}\left(\frac{\alpha_{s}}{1-\alpha_{g}}\right)^{2 / 3} \\
B_{g j}=\mathscr{B}_{g j} \rho_{g} \frac{\left(1-\alpha_{g}\right)}{d_{p}}\left(\frac{\alpha_{s}}{1-\alpha_{g}}\right)^{1 / 3} \\
\text { for } j=[s, l]
\end{gathered}
$$

Model 2 - Lappalainen et al. [8]:

$$
\begin{aligned}
& A_{g j}= \mathscr{A}_{g j} \mu_{g} \frac{\left(1-\alpha_{g}\right)^{2}}{d_{p}^{2} \alpha_{g}} \\
& B_{g j}=\mathscr{B}_{g j} \rho_{g} \frac{\left(1-\alpha_{g}\right)}{d_{p}} \\
& \quad \text { for } j=[s, l]
\end{aligned}
$$

Model 3 - Fourati et al. [9]:

$$
\begin{gathered}
A_{g s}=\mathscr{A}_{g s} \mu_{g} \frac{\alpha_{g} \alpha_{s}^{2}}{d_{p}^{2}\left(1-\alpha_{s}\right)} \\
B_{g s}=\mathscr{B}_{g s} \rho_{g} \frac{\alpha_{g}^{2} \alpha_{s}}{d_{p}^{2}\left(1-\alpha_{s}\right)^{2}} \\
A_{g l}=\mathscr{A}_{g l} \mu_{g} \frac{\alpha_{s}^{2}}{d_{p} \alpha_{g}} \\
B_{g l}=\mathscr{B}_{g l} \rho_{g} \frac{\alpha_{s}}{d_{p}}
\end{gathered}
$$

The diameter of packing used in the three models can be given as [4]:

$$
d_{p}=6 \frac{\alpha_{s}}{a_{s}}
$$

Model 4 :

The final model bases the gas-liquid interaction on the increase in specific pressure loss when the packed bed is irrigated [10]. The gas-solid and liquid-solid interactions takes a form the resembles the Darcy-Forchheimer model and are given as:

$$
\begin{array}{r}
\vec{F}_{g s}=\alpha_{g}\left(\mathscr{A}_{g s} f_{A, g s} \mu_{g}+\mathscr{B}_{g s} f_{B, g s} \rho_{g}\left|\vec{u}_{g}\right|\right) \vec{u}_{g} \\
\vec{F}_{l s}=\alpha_{l}\left(\mathscr{A}_{l s} f_{A, l s} \mu_{l}+\mathscr{B}_{l s} f_{B, l s} \rho_{l}\left|\vec{u}_{l}\right|\right) \vec{u}_{l}
\end{array}
$$

It can be seen that the respective coefficients are scaled by a function $f$. These functions are dependent on the volume fraction of the phase in question and will be determined when calibrating the models.

Dividing the specific pressure loss as shown in Equation 26 allows for the determination of the gas-liquid interaction force:

$$
\begin{gathered}
\nabla p=\nabla p_{d r y}+\nabla p_{w e t} \\
\vec{F}_{g l}=\frac{\nabla p_{w e t}}{\left|\vec{u}_{l}-\vec{u}_{g}\right|}\left(\vec{u}_{l}-\vec{u}_{g}\right)
\end{gathered}
$$

From Equation 11 it can be inferred that the dry specific pressure loss can be given as:

$$
\nabla p_{d r y}=\vec{F}_{g s}
$$

\section{MODEL COMPARISON}

The model parameters are fitted to data using least-squares to minimise the error between the data and the model. Data for the packing material is found in the literature where Linek et al. [11] reports the liquid holdup and Svetoslav et al. [12] reports pressure losses at varying liquid loads and gas velocities for the packing IMTP-50. The specific surface area and volume fraction for the IMPT-50 packing are:

$$
a_{s}=100 \frac{m^{2}}{m^{3}} \quad \alpha_{s}=0.02
$$

\section{Gas-solid}

When the packing is dry $\alpha_{g}=1-\alpha_{s}$ and Equations 16, 18 and 20 can be reduced and becomes identical. Thus the only varying parameter in the three first models is the viscous model parameter $\mathscr{A}_{g s}$. Additionally the wetting factor $\eta=0$. The specific pressure loss for the calibrated the gas-solid interaction are shown in Figure 2.

In Table 1 the calibrated coefficients are presented. Here it can be seen that the viscous parameter for all models are close to zero. Considering that the contribution to the interaction force for these are scaled by the viscosity, they can be neglected without introducing any noticeable error to model predictions. Additionally, the functions $f_{A, g s}$ and $f_{B, g s}$ in Equation 24 for model 4 were found to be unity.

Table 1. Coefficients for the calibrated gas-solid interaction and function expression for model 4

\begin{tabular}{c|cc} 
& $\mathscr{A}_{g s}$ & $\mathscr{B}_{g s}$ \\
\hline model 1 & $1.990 \cdot 10^{-4}$ & $1.655 \cdot 10^{0}$ \\
model 2 & $1.486 \cdot 10^{-5}$ & $1.655 \cdot 10^{0}$ \\
model 3 & $1.486 \cdot 10^{-5}$ & $1.655 \cdot 10^{0}$ \\
model 4 & 0.000 & $2.874 \cdot 10^{1}$ \\
& & \\
& $f_{A, g s}$ & $f_{B, g s}$ \\
\hline model 4 & 1 & 1
\end{tabular}




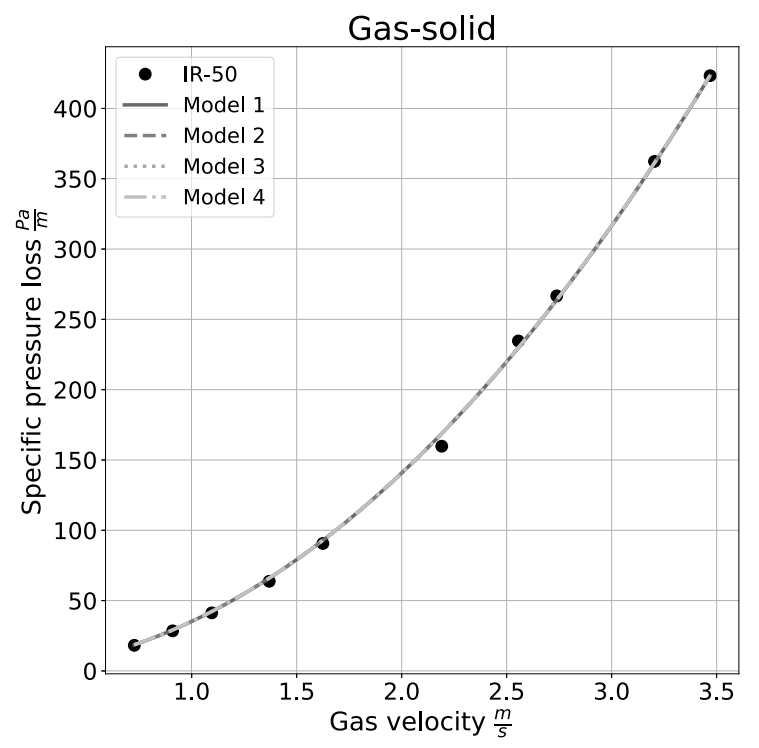

Figure 2. Specific pressure loss for the dry packing calculated by the models which are calibrated with data form Svetoslav et al. [12].

\section{Liquid-solid}

To model the wetting which is required for the liquid-solid interaction the parameter $C_{h}$ has to be calibrated to the packing material. A comprehensive list of values for $C_{h}$ is available [4]. However, it is not given for the IMPT packing which is studied here. To obtain a value for $C_{h}$ an expression for the liquid holdup that is dependent on the wetting factor is used [4]:

$$
\alpha_{l}=\left(12 \frac{\mu_{l}}{|\vec{g}| \rho_{l}} v_{l} a_{s}^{2}\right)^{1 / 3} \eta^{2 / 3}
$$

The expression given in Equation 29 is only valid below the loading point of the packing. It was found that for $C_{h}=1.1$ the liquid holdup is predicted with errors of $\pm 1-7 \%$ for liquid loads below $60 \mathrm{~m}^{3} / \mathrm{m}^{2} h$ whereas the error is $13 \%$ for a liquid load of $97 \mathrm{~m}^{3} / \mathrm{m}^{2} \mathrm{~h}$. The resulting predictions for the liquid holdup for Equation 29 and the calibrated liquid-solid interactions are shown in Figure 3.

It can be observed in Figure 3 the wetting factor has little influence on the liquid holdup predicted by models 1-3. The coefficients for these models were found not to change noticeably when the wetting factor was not accounted for. Moreover, model 1-3 does not represent the data for the liquid holdup accurately compared to model 4 . By examining the calibrated coefficients shown in Table 2 it can be seen the viscous coefficient $\mathscr{A}_{l s}$ is small for all of the porosity models. Therefore, it has little influence on the interaction force as is the case for gas-solid interaction. For model 4 the function $f_{B, l s}$ were found to be the squared inverse of the liquid holdup. Whereas for model 1-3 there is no dependency on the liquid holdup as it can be seen in Equation 14 which causes the poor performance of models $1-3$.

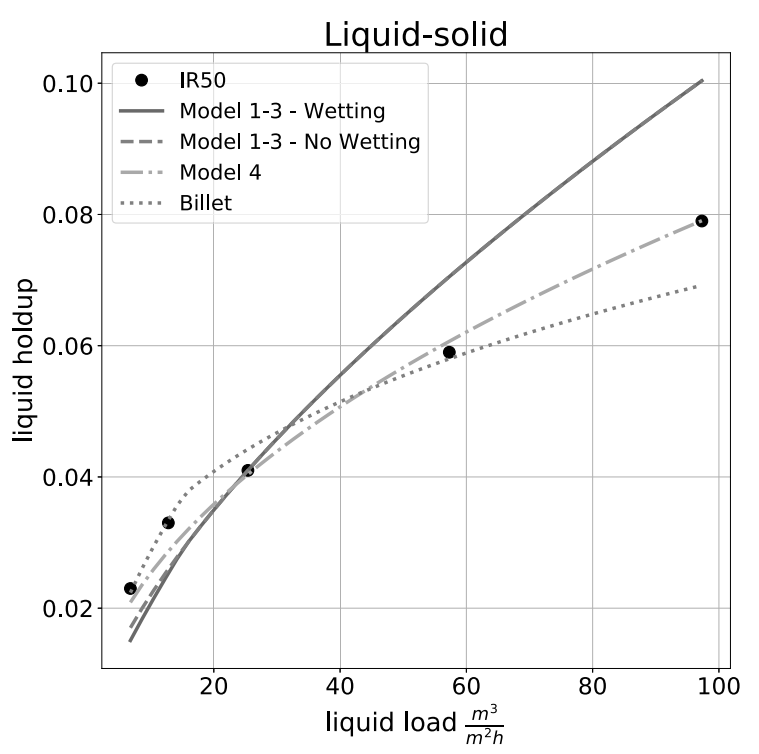

Figure 3. Liquid holdup in the packing with stagnant gas predicted by the models which are calibrated with data from Linek et al. [11].

Table 2. Coefficients for the calibrated liquid-solid interaction and function expression for model 4

\begin{tabular}{|c|c|c|}
\hline & $\mathscr{A}_{l s}$ & $\mathscr{B}_{l s}$ \\
\hline \multirow{3}{*}{$\begin{array}{l}\text { models } 1-3 \\
\text { model } 4\end{array}$} & $3.718 \cdot 10^{-3}$ & $8.149 \cdot 10^{-1}$ \\
\hline & 0.000 & $5.244 \cdot 10^{-1}$ \\
\hline & $f_{A, l s}$ & $f_{B, l s}$ \\
\hline model 4 & 1 & $\alpha_{l}^{-2}$ \\
\hline
\end{tabular}

\section{Gas-Liquid}

In the data set used for calibrating the gas-liquid interactions the liquid holdup was not measured. Instead the liquid holdup estimated by the liquid-solid interaction is utilised for the respective models. Data for other packing types and the data used for the liquid-solid interaction shows that the gas velocity has little influence on the liquid holdup as long as the packing is operated below the loading point $[3,11]$.

It can be seen from Figure 4 that the models 1-3 again cannot approximate the data no matter if the wetting factor is applied or not. However models 1 and 2 consistently over estimates the specific pressure loss when the wetting factor is not applied. Model 3 shows some improvement compared to model 1 and 2 when the wetting factor is not applied but an increase in the liquid load does not produce the increase in specific pressure loss seen in the data. It is notable that for models 1-3 the error between predicted pressure loss and the data decreases significantly at higher liquid loads. The mean absolute error between the specific pressure loss predicted at $10 \mathrm{~m}^{3} / \mathrm{m}^{2} \mathrm{~h}$ and $120 \mathrm{~m}^{3} / \mathrm{m}^{2} \mathrm{~h}$ for models $1-3$ are $27.7-17.7 \%, 30.4-18.5 \%$ and $40.8-18.0 \%$, respectively. 

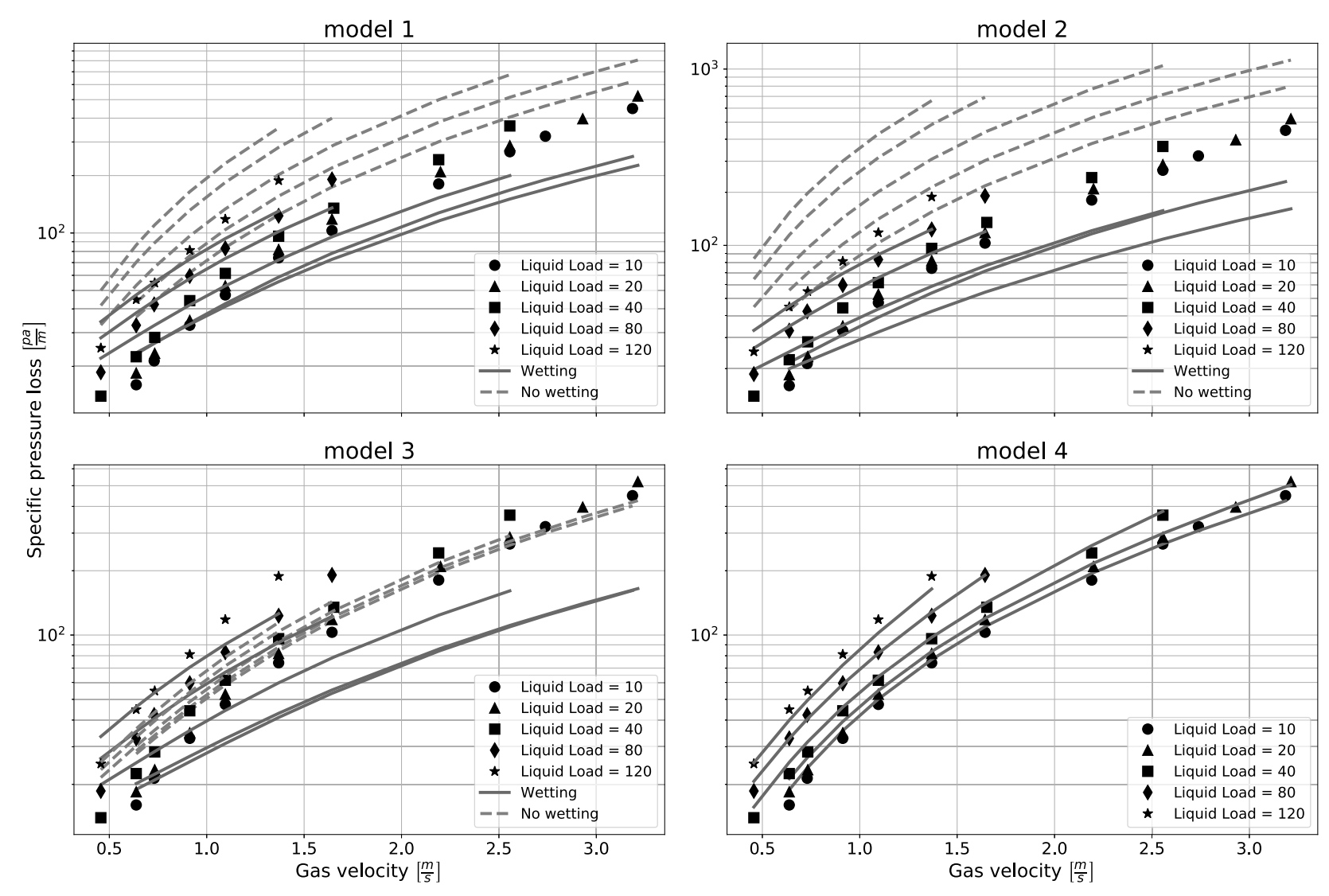

Figure 4. Specific pressure loss in the packing with liquid loads varying from 10-120 $\frac{\mathrm{m}^{3}}{m^{2} h}$. The models are calibrated with data from Svetoslav et al. [12].

As for the gas-solid and liquid solid interactions it can be seen from the calibrated coefficients in Table 3 that the viscous model coefficient $\mathscr{A}_{g s}$ can be neglected for all models. The values found for $\mathscr{A}_{g s}$ where all in the order of $10^{-11}$ and are therefore reported as 0 .

Table 3. Coefficients for the calibrated gas-liquid interactions

\begin{tabular}{c|cc} 
& $\mathscr{A}_{g s}$ & $\mathscr{B}_{g s}$ \\
\hline model 1 & $0.000 \cdot 10^{0}$ & $4.079 \cdot 10^{-1}$ \\
model 2 & $0.000 \cdot 10^{0}$ & $2.028 \cdot 10^{-1}$ \\
model 3 & $0.000 \cdot 10^{0}$ & $2.078 \cdot 10^{-1}$
\end{tabular}

As previously mentioned the increase in pressure loss at a given gas velocity when the packing is irrigated is caused by the increase in liquid film thickness. Consequential, the specific wet pressure loss should be dependent on the gas velocity and the liquid holdup. The expression found for the specific wet pressure loss used in model 4 are given below:

$$
\nabla p_{\text {wet }}=92.11 \alpha_{l} e^{\alpha_{l}\left(12.39 \alpha_{l}+0.695\right)\left|\vec{u}_{g}\right|}
$$

Using this expression in Equation 26 results in an average absolute error for the entire data set of $6.7 \%$ and a maximum error of $17.7 \%$ for a single data point. The largest relative errors are seen at gas velocities below $1 \mathrm{~m} / \mathrm{s}$ especially when the liquid load is between 10 and $40 \mathrm{~m}^{3} / \mathrm{m}^{2} \mathrm{~h}$.
Generally the expression tends to over predict the specific pressure loss for all liquid loads except $120 \mathrm{~m}^{3} / \mathrm{m}^{2} \mathrm{~h}$, where the model under predicts the specific pressure loss with $10 \%$ on average.

\section{SIMULATION}

Considering that the porosity models are calibrated using an idealised set of equations the performance is evaluated in a CFD simulation. As porosity models 1-3 were shown not to perform well for the IMTP packing material they will not be tested going further.

The solver multiphaseEulerFoam from OpenFOAM v8.0 is used to carry out simulations. Here the phases are treated as isothermal with constant densities and viscosities. The properties of the gas and liquid phases are set to that of air and water at $20^{\circ} \mathrm{C}$, respectively. The packing material is considered as a stationary phase, meaning that no momentum or transport equations are solved for this phase and that its velocity is zero. The stationary phase is essentially a place holder for the volume fraction equation and the interaction force equations. This is to ensure that the sum of the gas and liquid volume fractions are that of the bed porosity and to simplify the implementation of the porosity models.

The simulations are setup for a cylinder with height of $5 \mathrm{~m}$ and a radius of $0.4 \mathrm{~m}$. At the bottom a liquid outlet and 
a gas inlet is set and vice versa for the top. The packed bed is centred in the cylinder with a height of $3 \mathrm{~m}$. Utilising the symmetry of the cylinder a 2D axisymmetric mesh with a wedge boundary condition is used to simplify the case. To evaluate the specific pressure loss and liquid holdup in the packed bed the pressure gradient and liquid volume faction are volume averaged over its entirety. The specific pressure loss is corrected according to Equation 3, where the gravitational contribution is subtracted from volume averaged gradient.

In Figure 5 the results of the CFD model is compared with the data. It can be seen that the errors of the model has increased which is caused by the assumption of constant liquid holdup made for the gas-liquid interaction force. In the model results it is seen that the liquid holdup differs from that used in the model calibration by as much as $15 \%$ when the gas velocity is above $1 \mathrm{~m} / \mathrm{s}$. The mean absolute error for the entire data set has increased to $10.5 \%$ with a maximum error of $26.4 \%$ for a single data point.

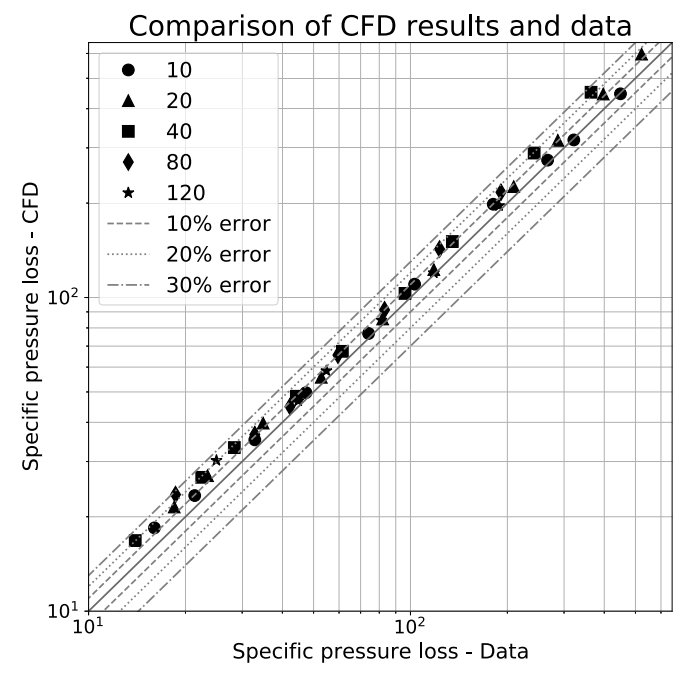

Figure 5. Comparison of CFD model results and data from Svetoslav et al. [12]

\section{CONCLUSION}

Four different porosity models have been tested and compared on their predictive performance with respect to the high capacity random packing material IMTP-50.

It was found that all models were independent on the viscous parameter when calibrated to the data. All models were found to match data for the gas-solid interaction without any noticeable errors. Models 1-3 were found to be unable to represent the data with respect to both liquid holdup and specific pressure loss. This was found to be the case whether or not the wetting factor was as applied to the interaction forces. Model 4 was found to predict the liquid holdup with reasonable accuracy. For the specific pressure loss the average error model was found to be $6.7 \%$ for the entire data set with a small bias towards over prediction. However for a liquid load of $120 \mathrm{~m}^{3} / \mathrm{m}^{2} \mathrm{~h}$ the model was found to be biased towards under prediction of the specific pressure loss with and average error of $10 \%$.

Considering the poor performance of models 1-3 only model 4 was implemented in a CFD model. Here it was found that the mean absolute error increased from $6.7 \%$ for the idealised equations to $10.5 \%$ for the CFD model. This increase were found to be mainly caused by the assumption of the liquid holdup being independent of gas velocity when calibrating the gas-liquid interaction for the model.

\section{ACKNOWLEDGMENTS}

The authors would like to acknowledge the financial support from the Innovation Fund Denmark [8053-00053B] to carry out this work .

\section{References}

[1] J.L. Kang, Y.C. Ciou, D.Y. Lin, D.S.H. Wong, S.S. Jang, Chemical Engineering Research and Design 147, 43 (2019)

[2] Y. Fu, J. Bao, R. Singh, C. Wang, Z. Xu, Chemical Engineering Science 221, 115693 (2020)

[3] R. Billet, Packed Towers in Processing and Environmental Technology (VCH Verlagsgesellschaft $\mathrm{mbH}$, 1995), ISBN 3-527-28616-0

[4] R. Billet, M. Schultes, Chemical Engineering Research and Design 77, 498 (1999)

[5] S. Ergun, A.A. Orning, Industrial \& Engineering Chemistry 41, 1179 (1949)

[6] J.R. Finn, J.E. Galvin, Chemical Engineering Science 172, 722 (2017)

[7] A. Attou, C. Boyer, G. Ferschneider, Chemical Engineering Science 54, 785 (1999)

[8] K. Lappalainen, V. Alopaeus, M. Manninen, J. Aittamaa, Industrial \& Engineering Chemistry Research 47, 8436 (2008)

[9] M. Fourati, V. Roig, L. Raynal, Chemical Engineering Science 100, 266 (2013), 11th International Conference on Gas-Liquid and Gas-Liquid-Solid Reactor Engineering

[10] F.H. Yin, C.G. Sun, A. Afacan, K. Nandakumar, K.T. Chuang, Industrial \& engineering chemistry research 39, 1369 (2000)

[11] V. Linek, T. Moucha, F. Rejl, Chemical Engineering Research and Design 79, 725 (2001), distillation and Absorption

[12] N. Svetoslav, D. Dzhonova, N. Kolev, Bulgarian Chemical Communications 44, 283 (2012) 